\title{
Article \\ R2R3-MYB Transcription Factor PlMYB108 Confers Drought Tolerance in Herbaceous Peony (Paeonia lactiflora Pall.)
}

\author{
Yanqing $\mathrm{Wu}^{1,2}$, Tingting $\mathrm{Li}^{2}$, Zhuoya Cheng ${ }^{2}$, Daqiu Zhao ${ }^{2}$ and Jun Tao ${ }^{1,2, *}$ \\ 1 Joint International Research Laboratory of Agriculture and Agri-Product Safety, The Ministry of Education of \\ China, Institutes of Agricultural Science and Technology Development, Yangzhou University, \\ Yangzhou 225009, China; yqwu@yzu.edu.cn \\ 2 College of Horticulture and Plant Protection, Yangzhou University, Yangzhou 225009, China; \\ tingtingliyzu@163.com (T.L.); zhuoya.edu@outlook.com (Z.C.); dqzhao@yzu.edu.cn (D.Z.) \\ * Correspondence: taojun@yzu.edu.cn; Tel.: +86-514-87997219; Fax: +86-514-87347537
}

check for updates

Citation: Wu, Y.; Li, T.; Cheng, Z.; Zhao, D.; Tao, J. R2R3-MYB Transcription Factor PlMYB108 Confers Drought Tolerance in Herbaceous Peony (Paeonia lactiflora Pall.). Int. J. Mol. Sci. 2021, 22, 11884. https://doi.org/10.3390/ijms 222111884

Academic Editors:

Magdalena Arasimowicz-Jelonek,

Arkadiusz Kosmala,

Ewa Sobieszczuk-Nowicka and Ricardo Aroca

Received: 3 September 2021

Accepted: 26 October 2021

Published: 2 November 2021

Publisher's Note: MDPI stays neutral with regard to jurisdictional claims in published maps and institutional affiliations.

Copyright: (c) 2021 by the authors. Licensee MDPI, Basel, Switzerland. This article is an open access article distributed under the terms and conditions of the Creative Commons Attribution (CC BY) license (https:// creativecommons.org/licenses/by/ $4.0 /)$.

\begin{abstract}
The MYB transcription factor (TF) is crucial for plant growth, development, and response to abiotic stress, but it is rarely reported in the herbaceous peony (Paeonia lactiflora Pall.). Here, an MYB TF gene was isolated, and based on our prior mRNA data from P. lactiflora samples, it was treated with drought stress (DS). Its complete cDNA structure was 1314 bp, which encoded 291 amino acids (aa). Furthermore, using sequence alignment analysis, we demonstrated that PIMYB108 was an R2R3-MYB TF. We also revealed that PIMYB108 was primarily localized in the nucleus. Its levels rose during DS, and it was positively correlated with drought tolerance (DT) in P. lactiflora. In addition, when PIMYB108 was overexpressed in tobacco plants, the flavonoid content, antioxidant enzyme activities, and photosynthesis were markedly elevated. Hence, the transgenic plants had stronger DT with a higher leaf water content and lower $\mathrm{H}_{2} \mathrm{O}_{2}$ accumulation compared to the wildtype (WT) plants. Based on these results, PIMYB108 is a vital gene that serves to increase flavonoid accumulation, reactive oxygen species (ROS), scavenging capacity, and photosynthesis to confer DT. The results would provide a genetic resource for molecular breeding to enhance plant DT.
\end{abstract}

Keywords: MYB; drought stress; flavonoid; ROS; Paeonia lactiflora

\section{Introduction}

As the global climate continues to warm, a large quantity of water evaporates, resulting in a massive reduction in soil water content and subsequent drought conditions [1]. Being a major abiotic stressor, drought severely affects growth, development [2], yield [3], and quality [4] of plants, which in turn negatively affects agricultural supply. In China, the northern regions are mostly dry or semidry. As a result, in these areas, drought remains a major factor restricting landscaping construction. Therefore, the cultivation of droughttolerant plant varieties and the generation of a low water consumption model and an esthetic plant configuration model are the main directions of green construction in the northern region.

Plants have evolved a variety of survival mechanisms in order to adapt to arid environments. One such mechanism is the regulation of drought-resistant genes [5]. Droughtresistant genes are mainly divided into two types: functional and regulatory. Transcription factors (TFs) belong to regulatory genes, which modulate the levels of multiple functional genes related to drought resistance. Multiple studies revealed that this is the most effective approach to enhancing plant drought resistance [6]. At present, the TF families associated with plant drought resistance mainly include bZIP (Basic leucine zipper), AP2/ERF (APETALA2/Ethylene Response Element Binding Factors), MYB (myeloblastoma), WRKY, NAC, etc. [7-9]. Of this list, MYB is the most prevalent in plants [10]. Its $N$-terminus has a highly conserved MYB DNA-binding domain, and it encodes proteins with 51-53 amino acid (aa) [11]. The MYB TFs are further categorized into four groups, 
namely, 1R-MYB/MYB-related, R2R3-MYB, 3R-MYB (R1R2R3-MYB), and 4R-MYB based on the number of unit repetitions within the MYB domain [12,13]. Among them, the R2R3-MYB TFs contribute greatly to plant growth and development [14] and abiotic stress response [15,16]. In a study by Zhang et al. [17], it was reported that the wheat gene R2R3-MYB TF TaMYB30-B improved drought stress (DS) tolerance in transgenic Arabidopsis. Similarly, the poplar R2R3-MYB TF PtrMYB94 modulates abscisic acid signaling to enhance drought tolerance (DT) in plants [18]. These studies indicate that the R2R3-MYB TF can be used as an important target for improving drought resistance in plants.

The herbaceous peony (Paeonia lactiflora Pall.) is a perennial herbaceous flower that can survive for long periods of time and can massively reduce landscaping costs. P. lactiflora has a long history of cultivation in China, and it is mainly cultivated in the northern regions. In these regions, however, drought is a major stress factor affecting the cultivation of P. lactiflora. In P. lactiflora, the physiological and biochemical responses under drought stress were investigated [19], and drought stress changed the expression of differentially expressed genes (DEGs) involved in the reactive oxygen species (ROS) system, chlorophyll degradation and photosynthetic capacity, secondary pathways of biosynthesis, and sugar metabolism [20]. However, studies involving the TFs that regulate P. lactiflora DT are rarely reported. Herein, an R2R3-MYB TF PlMYB108 was extracted from P. lactiflora, and its function in conferring DT was examined in transgenic tobacco plants.

\section{Results}

\subsection{Extraction and Sequence Analysis of PlMYB108}

Based on our previous transcriptome data of $P$. lactiflora treated with drought stress, (SAR: SRP131648) [20], the TF MYB108 (Unigene0031519), produced the largest difference from the controls; we examined its full-length cDNA via RACE. In addition, the result demonstrates that MYB108 has an open reading frame (ORF) of $876 \mathrm{bp}$, a $5^{\prime}$ UTR (untranslated region) of $156 \mathrm{bp}$ and a 3' UTR of $282 \mathrm{bp}$. Moreover, it encodes a 291 aa protein (Figure 1).

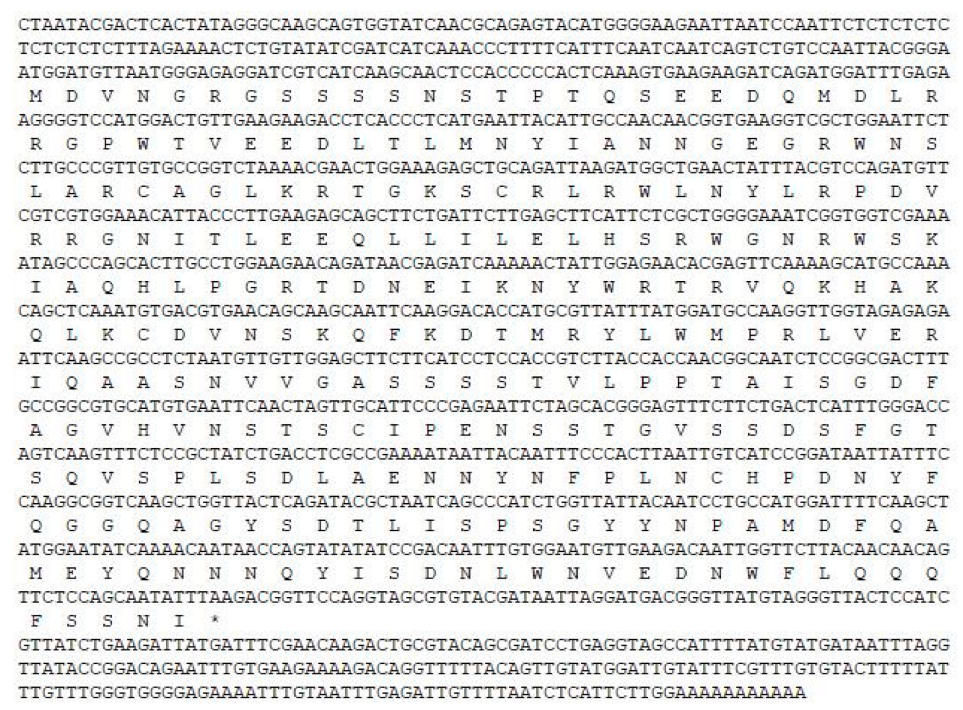

Figure 1. cDNA sequence of PIMYB108 and its deduced amino acid sequence. The deduced amino acid sequence is shown underneath the corresponding nucleotide sequence, the others are noncoding regions, and the stop code was indicated with *.

Using the web software ProtParam, we further revealed that PIMYB108 has a molecular weight of 33,009.52 Da and a theoretical isoelectric point (pI) of 6.22. Based on this evidence, we identified this protein to be both unstable and hydrophilic. Moreover, using the web softwares TMHMM and SignalP 5.0, we estimated that PIMYB108 had no signal 
peptide and transmembrane sites. This suggests that PIMYB108 is likely a nonsecretory and/or nontransmembrane protein.

The homologous sequence alignments of PIMYB108 with Populus alba MYB108 (XP_034 907007), Populus trichocarpa MYB108 (XP_002316060), Populus euphratica MYB108 (XP_01101 0946), Paeonia suffruticosa MYB13 (QIG55696), Camellia sinensis MYB108 (XP_028115735), Vitis vinifera MYB108 (RVW26470), Salix suchowensis MYB (KAG5237142), Arabidopsis thaliana MYB108 (NP_187301), Arabidopsis thaliana MYB111 (NP_199744), and Arabidopsis thaliana MYB112 (NP_564519) were performed by DNAMAN5.2.2, and they shared a homology rate of $62.39 \%$. Based on a sequence analysis, PIMYB108 was shown to contain both $N$-terminal R2 and R3 MYB DNA-binding domains, thereby suggesting that it is an R2R3-MYB TF (Figure 2A). Meanwhile, we also generated a phylogenetic tree via the Neighbor-Joining method (NJ) and MEGA5.05. Additionally, we followed numerous protein sequence alignments using ClustalW. Together, the data demonstrate that PIMYB108 is strongly correlated with PsMYB13 (QIG55696) (Figure 2B).

A

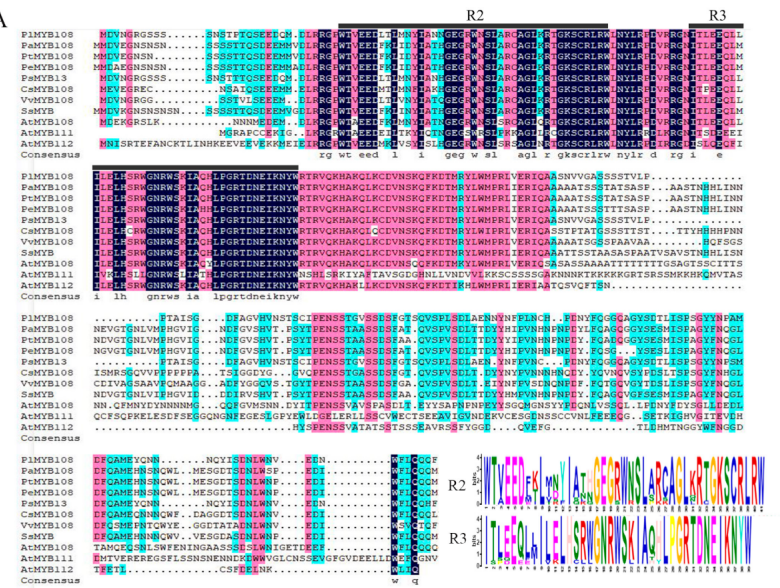

B

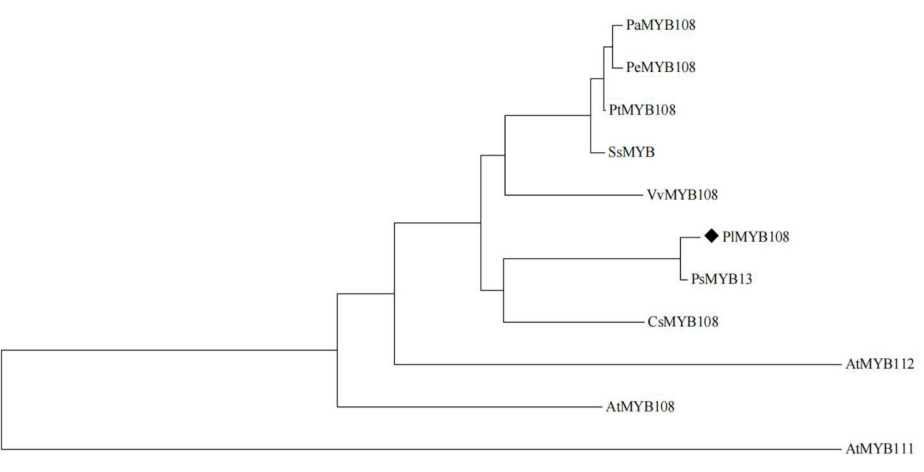

Figure 2. Sequences alignment and phylogenetic tree of PIMYB108 and seven other MYB proteins from plants. (A) Sequences alignment. The R2 and R3 MYB domains shown refer to two repeats of the MYB DNA-binding domain of MYB proteins. (B) Phylogenetic tree. Protein accession numbers are as follows: Populus alba MYB108 (XP_034907007), Populus trichocarpa MYB108 (XP_002316060), Populus euphratica MYB108 (XP_011010946), Paeonia suffruticosa MYB13 (QIG55696), Camellia sinensis MYB108 (XP_028115735), Vitis vinifera MYB108 (RVW26470), Salix suchowensis MYB (KAG5237142), Arabidopsis thaliana MYB108 (NP_187301), Arabidopsis thaliana MYB111 (NP_199744), and Arabidopsis thaliana MYB112 (NP_564519).

\subsection{PIMYB108 Expression Pattern Analysis in P. lactiflora Exposed to DS}

The PlMYB108 expression pattern was determined in P. lactiflora exposed to DS (Figure 3). With increasing duration, the PIMYB108 levels increased remarkably in controls, whereas under natural DS, the PlMYB108 levels still increased, reaching their highest expression on the 21st day post exposure. Relative to the controls, the PlMYB108 levels in P. lactiflora exposed to DS were markedly high. 


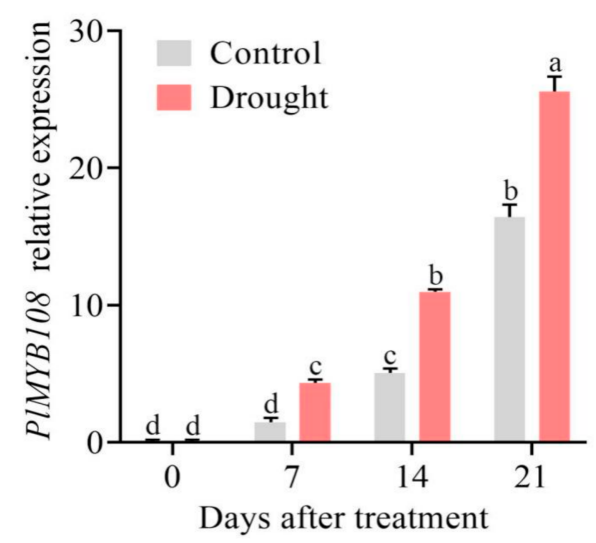

Figure 3. Relative expression levels of PIMYB108 in P. lactiflora under drought stress. The values represent the mean $\pm S D$, and different letters indicate significant differences according to Duncan's multiple range test $(p<0.05)$.

\subsection{Examining Localization of the PlMYB108 Protein}

To examine the localization of the PIMYB108 protein, the constructed plasmid p35S:PlM YB108-GFP was transformed in tobacco leaves. Based on our data, the emitted fluorescence is strongly expressed in the nucleus, which indicates that PIMYB108 is primarily a nuclear protein (Figure 4).

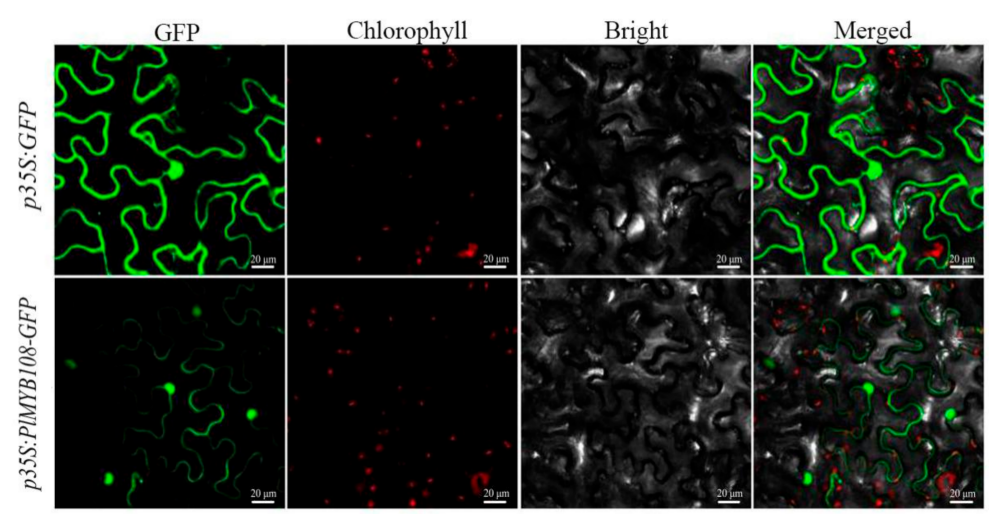

Figure 4. Subcellular localization of PIMYB108 in tobacco leaves. Note: From left to right are superimposed photos of GFP (green fluorescent protein), chloroplast autofluorescence, bright field, and three channels of target gene/empty vector, respectively.

\subsection{PlMYB108 Conferred DS Tolerance in Tobacco}

PCR and qRT-PCR were used for the identification of transgenic tobacco plants in this study. After 2 months of cultivation, the leaves of all the tobacco plants were employed as materials for identification. The PCR analysis revealed that all the tobacco plants produced a single bright band of NtActin, and the products of PlMYB108 were detected only in the transgenic lines (TL) but not in WT (Figure 5A). In addition, we performed a further analysis using qRT-PCR. We demonstrated that the PIMYB108 levels in TL were markedly elevated relative to WT (Figure 5B). These results suggest that PIMYB108 was successfully transformed in tobacco plants. 
A

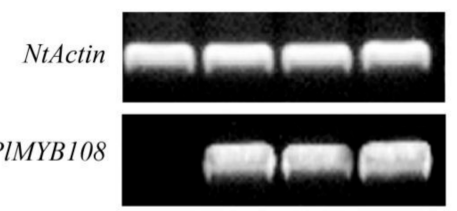

WT Line1 Line2 Line3

B

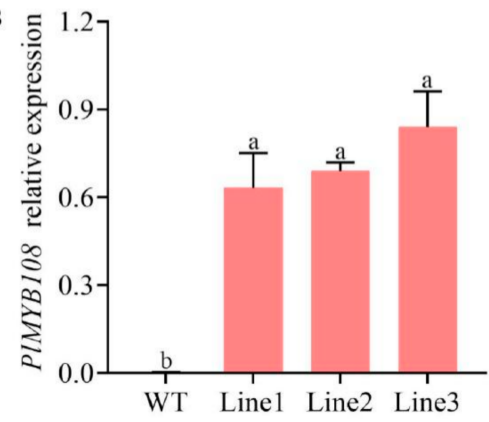

Figure 5. Identification of transgenic tobacco lines. (A) PCR analysis of PIMYB108 mRNA; (B) Relative expression level of PIMYB108 in leaves using qRT-PCR; the values represent the mean \pm SD, and different letters indicate significant differences according to Duncan's multiple range test $(p<0.05)$.

Next, the tobacco plants were exposed to natural DS for 10 days. Based on our results, the leaves of WT and TL showed massive differences. The WT leaves exhibited obvious wilting and drooping, whereas the TL ones exhibited sustained growth (Figure 6A). We also evaluated the relative leaf water content (RLWC) and $\mathrm{H}_{2} \mathrm{O}_{2}$ accumulation to assess the growth status of the TL post DS. Based on our results, the TL exhibited markedly increased RLWC and lower $\mathrm{H}_{2} \mathrm{O}_{2}$ accumulation when compared to the WT (Figure 6B,C).
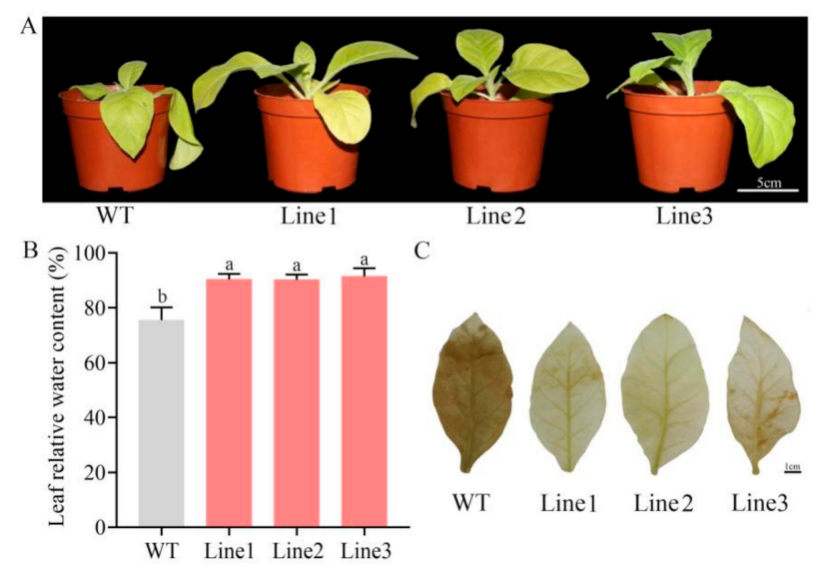

Figure 6. Phenotype and stress physiological indices of WT and transgenic lines under drought stress. (A) Phenotype of WT and transgenic lines under drought stress; (B) Relative leaf water content; (C) $\mathrm{H}_{2} \mathrm{O}_{2}$ accumulation was detected by DAB staining. Tobacco plants were stressed by drought for 10 days. The values represent the mean $\pm S D$, and different letters indicate significant differences according to Duncan's multiple range test $(p<0.05)$.

\subsection{PlMYB108 Increased Flavonoid Accumulation and Antioxidant Enzyme Activities}

To clarify how PlMYB108 confers DS tolerance within tobacco plants, the flavonoid content and antioxidant enzyme activities were assessed. As shown in Figure 7, the flavonoid content was markedly elevated $(\sim 40.52 \%)$ in TL compared to WT. Moreover, the antioxidant enzyme activities, including superoxide dismutase (SOD, EC 1.15.1.1), peroxidase (POD, EC 1.11.1.7), catalase (CAT, EC 1.11.1.6), and ascorbate peroxidase (APX, EC 1.11.1.11) were markedly increased, with values that were respectively $1.09,1.63,1.40$, and 1.43 times that of WT (Figure 8). 


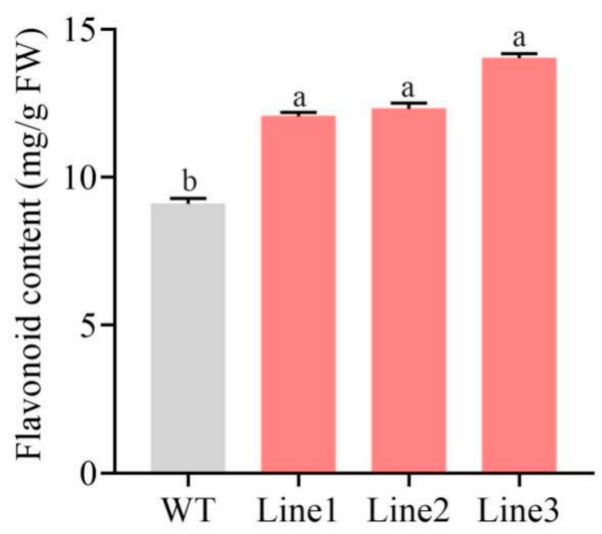

Figure 7. Flavonoid content of WT and transgenic lines under drought stress. Tobacco plants were stressed by drought for 10 days. The values represent the mean $\pm S D$, and different letters indicate significant differences according to Duncan's multiple range test $(p<0.05)$.
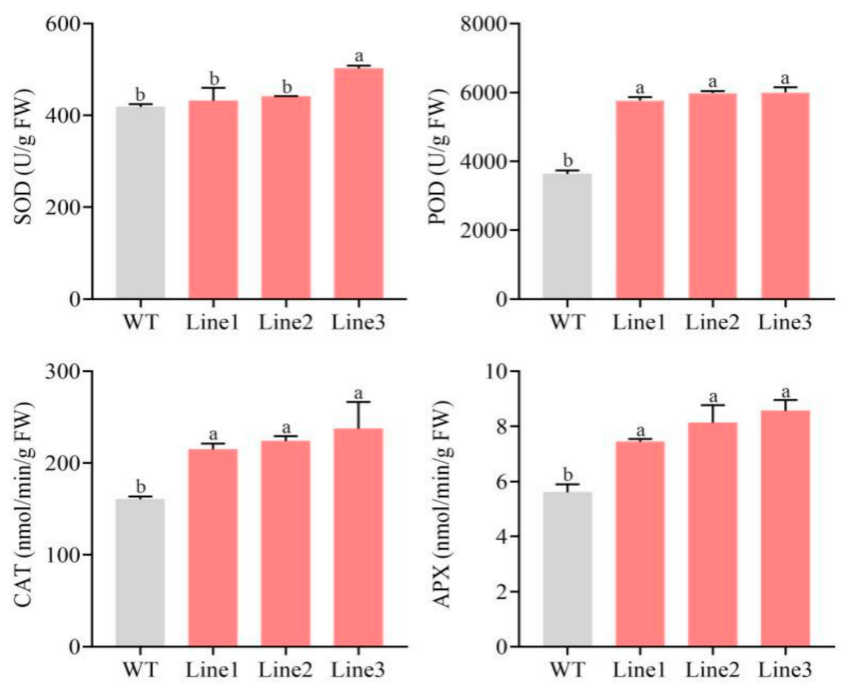

Figure 8. Antioxidant enzyme activities of WT and transgenic lines under drought stress. Tobacco plants were stressed by drought for 10 days. The values represent the mean $\pm \mathrm{SD}$, and different letters indicate significant differences according to Duncan's multiple range test $(p<0.05)$.

\subsection{PlMYB108 Enhanced Photosynthesis}

Subsequently, the photosynthetic characteristics, including net photosynthesis rate $(P n)$, stomatal conduction $(\mathrm{Gs})$, intercellular $\mathrm{CO}_{2}$ concentration $(\mathrm{Ci})$, and transpiration rate ( $(\mathrm{r})$ were analyzed. Compared to WT, Pn, Gs, Ci, and $\operatorname{Tr}$ increased by $14.98 \%, 12.44 \%$, $16.56 \%$, and $24.06 \%$ in TL, respectively (Figure 9 ). Additionally, the chlorophyll fluorescence parameters, including the minimum fluorescence (Fo), the maximum fluorescence (Fm), the non-photochemical quenching (qN), the actual photosynthetic efficiency of light system II $(\mathrm{Y}(\mathrm{II}))$, the variable fluorescence to maximum fluorescence ratio $(\mathrm{Fv} / \mathrm{Fm})$, and the variable fluorescence to minimum fluorescence $(\mathrm{Fv} / \mathrm{Fo})$ ratio, were also measured. Compared to WT, Fm, Y(II), Fv / Fm, and Fv/Fo were all remarkably elevated in TL compared to WT. Moreover, qN was slightly higher, while Fo was significantly lower (Figure 10), in TL than in WT. 

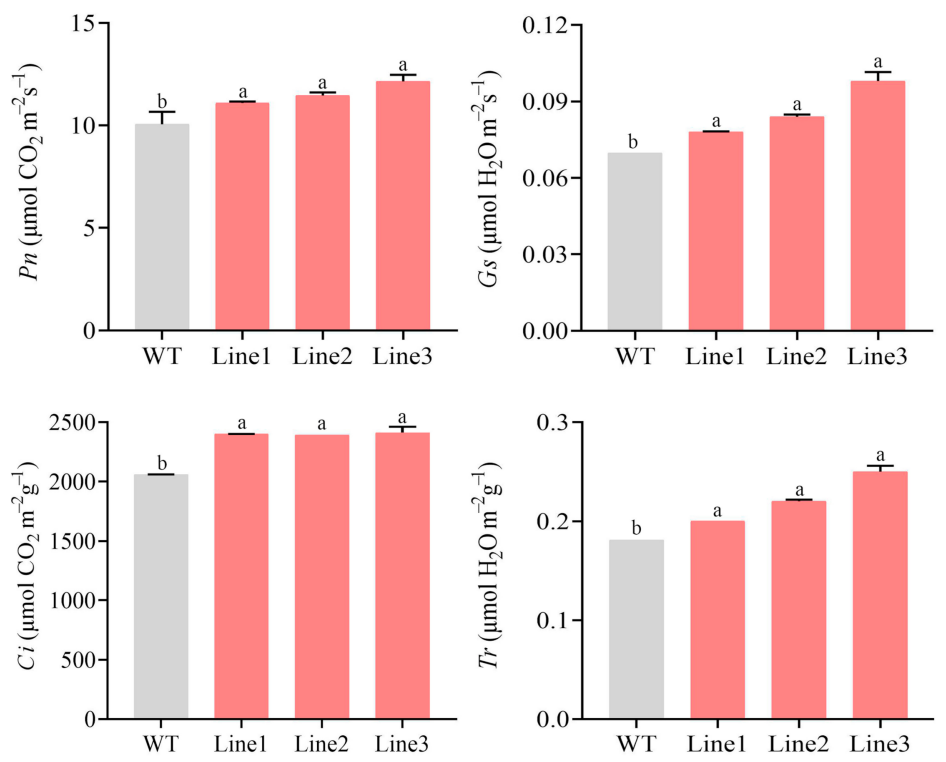

Figure 9. Photosynthetic characteristics of WT and transgenic lines under drought stress. Tobacco plants were stressed by drought for 10 days. The values represent the mean $\pm \mathrm{SD}$, and different letters indicate significant differences according to Duncan's multiple range test $(p<0.05)$.
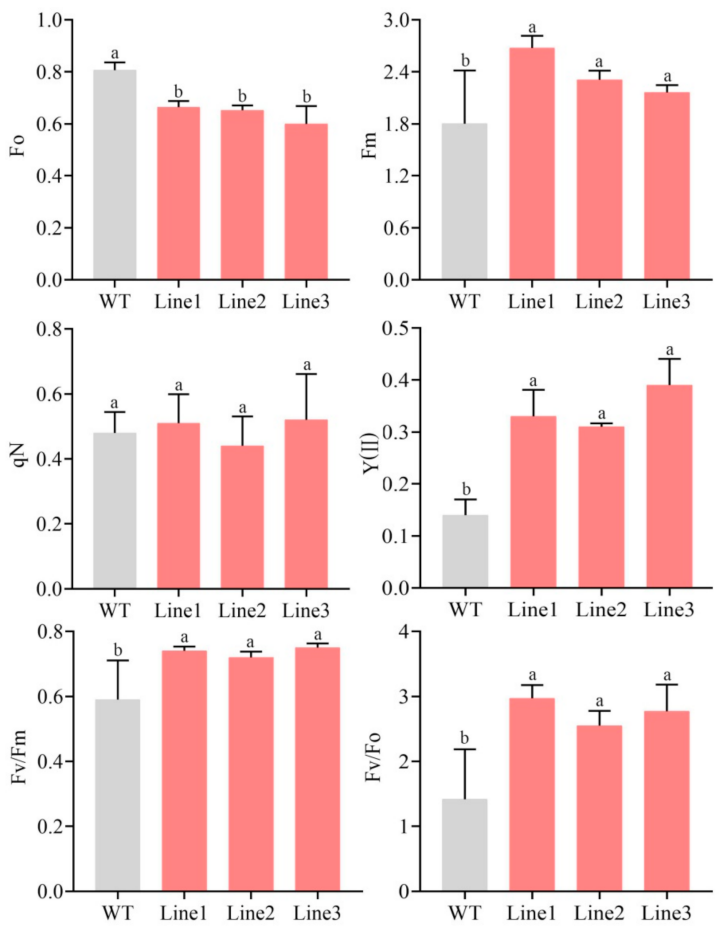

Figure 10. Chlorophyll fluorescence parameters of WT and transgenic lines under drought stress. Tobacco plants were stressed by drought for 10 days. The values represent the mean $\pm S D$, and different letters indicate significant differences according to Duncan's multiple range test $(p<0.05)$.

\section{Discussion}

Water shortage is a major problem facing the world today, and many garden flowers cannot develop under drought conditions. Therefore, cultivating drought-tolerant varieties is of great significance for landscaping in arid conditions. Multiple factors modulate plant drought resistance. When plants are drought-stressed, they resist and adapt to drought in different ways [21]. However, TFs that modulate the expression of genes related to plant resistance and stress have gained much attention [6]. MYB is among the highly prevalent 
TF families in plants [10]. Among them, the R2R3-MYB subtype is widely expressed and contributes greatly to plant growth and development [14] and response to abiotic stress $[15,16]$. At present, the R2R3-MYB TFs have been cloned in numerous plants. For example, Wang et al. [22] isolated a strawberry R2R3-MYB TF FvMYB24 that possessed the full-length coding region and encoded an estimated protein of 284 aa. Similarly, Zhang et al. [23] isolated PmMYBa1 from Prunus mume, which carried a conserved R2R3 MYB domain and belonged to the anthocyanin-associated subgroup 6 of the R2R3-MYB family. In this study, the full-length PIMYB108 cDNA was retrieved via RACE technology, which encoded a protein of 291 aa. Furthermore, using sequence analysis, we demonstrated that PIMYB108 carried both the N-terminal R2 and R3 MYB DNA-binding domains. This suggests that PIMYB108 is an R2R3-MYB TF with a high homology with MYB from other plants, as reported in a prior study [24].

In recent years, there have been extensive studies on the function of $M Y B$ in plant abiotic stress response. For example, Qin et al. [25] found that the expression of TaMYB33 in wheat leaves was induced by $\mathrm{NaCl}, \mathrm{PEG}$, and ABA treatments. Likewise, Wu et al. [26] also showed that PEG6000, $\mathrm{NaCl}$, and ABA treatments upregulated $\mathrm{ZmMYB3R}$ levels in maize. These results suggest that TaMYB33 and ZmMYB3R might modulate plant stress response. Here, we detected PlMYB108 levels using qRT-PCR. Under natural DS, PlMYB108 levels were markedly upregulated in P. lactiflora, which might positively correlate with DT in P. lactiflora. Subsequently, we investigated the subcellular localization of PIMYB108 and found that it primarily resided in the nucleus, which mirrors the RhMYB108 study in rose plants [27]. Based on this evidence, PIMYB108 is a nuclear protein, which might serve as a $\mathrm{TF}$ that plays a regulatory role in the nucleus.

A previous study reported cloning TaPIMPI, with MYB-typical characteristics, based on the homologous sequence of Arabidopsis AtMYB108. Furthermore, its overexpression in wheat markedly enhanced drought resistance in plants [28]. To further confirm the DT activity of PlMYB108 in P. lactiflora, PlMYB108 was incorporated into a model tobacco plant, as evidenced by PCR and qRT-PCR. Next, the tobacco plants underwent natural DS for 10 days. Following this, the WT leaves showed significant wilting and drooping, whereas the TL remained normal in its development. This phenotypic difference strongly suggests that PlMYB108 confers DT in tobacco. Moreover, relative leaf water content and $\mathrm{H}_{2} \mathrm{O}_{2}$ accumulation were markedly elevated in the TL compared to WT, which revealed that PlMYB108 can effectively maintain a normal water requirement and remove ROS.

Flavonoids represent a class of secondary metabolites that exert either positive or negative effects on plant growth. Nakabayashi et al. reported that overexpressing AtMYB12 and AtMYB75 in Arabidopsis remarkably enhanced DT via the overaccumulation of flavonoids [29]. In P. lactiflora, DS significantly increased flavonoid accumulation [20]. Moreover, in our study, the flavonoid content in TL was markedly increased $(40.52 \%)$ compared to WT. This, in turn, exerted positive effects on the DS of tobacco plants. In addition, antioxidant enzyme activities in TL were significantly elevated in our study relative to WT, which, in turn, increased the ROS scavenging capacity and reduced $\mathrm{H}_{2} \mathrm{O}_{2}$ accumulation.

Photosynthesis provides both energy and material resources for plants. In this study, $P n, G s, C i$, and $T r$ were markedly increased in TL versus WT. This suggests that PlMYB108 alleviates the DS-mediated suppression of photosynthesis, which is in accordance with results from the P. ostii caffeoyl-CoA O-methyltransferase overexpression examinations [30]. In addition, chlorophyll fluorescence parameters are used as internal indices of plants adaptation to the eco-environment, and their changes during environmental stress can be used to judge the degree of damage to photosynthetic organs. In this study, Fm, Y(II), $\mathrm{Fv} / \mathrm{Fm}$, and Fv/Fo in TL were markedly elevated, relative to WT, and the value of Fv/Fm was approximately 0.75 , suggesting that DS caused little damage to the PSII reaction center in TL compared to WT. Therefore, the TLs were better at maintaining normal photosynthesis during DS. Our conclusions would provide a theoretical basis for future studies attempting to enhance DS tolerance in P. lactiflora via genetic engineering. 


\section{Materials and Methods}

\subsection{Extraction and Bioinformatic Analysis of PlMYB108}

Total P. lactiflora RNA isolation was done with a MiniBEST Plant RNA Extraction Kit (TaKaRa, Kyoto, Japan). The entire cDNA was retrieved via rapid-amplification of cDNA ends (RACE) using a $3^{\prime}$ full RACE Core Set v2.0 (TaKaRa, Kyoto, Japan) and a SMARTer ${ }^{\mathrm{TM}}$ RACE cDNA Amplification Kit (Clontech, Mountain View, CA, USA) with targeted primers (Table S1). The PCR product was then separated on an agarose gel, excised, and sent for sequencing.

The aa composition, protein molecular weight, $\mathrm{pI}$, stability, and hydropathicity were estimated via the ProtParam (http:/ / web.expasy.org/protparam/, accessed on 20 August 2021) software. The transmembrane domain was analyzed via the TMHMM server 2.0 software (http:/ / www.cbs.dtu.dk/services/TMHMM, accessed on 20 August 2021). The signal peptide was estimated via the SignalP 5.0 Server (http:/ / www.cbs.dtu.dk/services/ SignalP /, accessed on 20 August 2021). The homologous PIMYB108 sequence alignment with other species was done via DNAMAN5.2.2, and the phylogenetic tree was generated via NJ of MEGA5.05.

\subsection{Expression Pattern Analysis of PlMYB108 P. Lactiflora Exposed to DS}

Five-year-old P. lactiflora plants in potting soil (loam:peat:perlite, 1:1:1) were employed for the DS-stimulated PIMYB108 expression pattern analysis. The P. lactiflora plants were placed in a glass greenhouse on the Wenhui Road campus of Yangzhou University. They were separated into 2 groups, which each contained six plants: one group received water at 17:00 daily and served as the control group, and the other group received natural DS. The top leaves were collected at $0,7,14$, and 21 days post exposure for an assessment of the PlMYB108 levels, using qRT-PCR via the BIO-RAD CFX Connect ${ }^{\mathrm{TM}}$ Optics Module (Bio-Rad, Hercules, CA, USA). All values were computed via the $2^{-\Delta \Delta C t}$ relative threshold cycle $(\mathrm{Ct})$ method [31]. Primer sequences are summarized in Table S2.

\subsection{Subcellular PlMYB108 Localization}

This analysis was done using tobacco leaves and confocal laser microscopy (Nikon C2ER, Tokyo, Japan). The p35S:PlMYB108-GFP gene fusions were synthesized to retrieve the PlMYB108 product (Figure S1). The ORF of PlMYB108 was amplified using primers carrying BsmB I restriction sites (forward 5'-CAGTGGTCTCACAACATGGATGTTAATGGGAG AGG-3' ${ }^{\prime}$, reverse $5^{\prime}$-CAGTGGTCTCATACAAATATTGCTGGAGAACTGTT-3'), which were then digested and introduced to the expression vectors with T4 DNA ligase (TaKaRa, Kyoto, Japan) to produce a set of $p 35 S$ :PlMYB108-GFP fusions, which were then sequenced for further validation. Subsequent transformations of the $p 35 S$ :PlMYB108-GFP and the empty p35S:GFP vector were conducted as reported previously [32].

\subsection{PlMYB108 Overexpression in TL Tobacco}

The $p 35 S-P l M Y B 108$ plasmid was incorporated into the Agrobacterium tumefaciens strain EHA105 using the freeze-thaw procedure. In turn, the strains were incorporated into tobacco (Nicotiana tabacum ' $\mathrm{k} 326^{\prime}$ ) via the leaf disc transformation procedure. Plasmid transformation was then verified using PCR and qRT-PCR. The primers used are summarized in Table S3.

\subsection{DS Exposure Affecting PlMYB108 in TL Tobacco}

The TL and WT tobacco plants were maintained for 2 months prior to use in natural DS experiments. The TL tobacco plants contained three lines, each line contained six plants. The plants underwent DS (i.e., no water) for 10 days. Next, the photosynthetic characteristic and chlorophyll fluorescence parameters were measured, and essential physiological variables such as $\mathrm{RLWC}, \mathrm{H}_{2} \mathrm{O}_{2}$, flavonoid content, and antioxidant enzyme activities were assessed. Following this, the leaves were kept at $-80{ }^{\circ} \mathrm{C}$ with liquid nitrogen until further gene expression analyses. 


\subsection{Measurements of Physiological Indexes, Photosynthetic Characteristics, and Chlorophyll Fluorescence Parameters}

An oven (Shanghai Jinghong Laboratory Instrument Co., Ltd., Shanghai, China) and balance (Suzhou Science Instrument Co., Ltd., Suzhou, China) were used to evaluate the RLWC. In brief, the leaf weight was measured and documented as fresh weight (FW). Next, the leaves were heated to $105{ }^{\circ} \mathrm{C}$ for $5 \mathrm{~min}$ in an oven, then cooled to $65^{\circ} \mathrm{C}$ for $>2 \mathrm{~h}$. The dried leaf weight was weighed again and recorded as dry weight (DW). Lastly, the RLWC was computed as follows: $(\mathrm{FW}-\mathrm{DW}) / \mathrm{FW} \times 100 \%$.

Diaminobenzidine (DAB) staining was employed for the detection of hydrogen peroxide $\left(\mathrm{H}_{2} \mathrm{O}_{2}\right)$ accumulation. In short, leaves were submerged in $0.1 \mathrm{mg} / \mathrm{mL}$ DAB in $50 \mathrm{mM}$ Tris-acetate buffer ( $\mathrm{pH}$ 5.0) in the dark at $25^{\circ} \mathrm{C}$. Following a $24 \mathrm{~h}$ incubation, the samples were removed and boiled in $95 \%(v / v)$ ethanol for $\geq 15 \mathrm{~min}$, before capturing images with a camera (Canon 50D, Tokyo, Japan).

The flavonoid content and antioxidant enzyme activities, namely SOD, POD, CAT, and APX, were assessed with reagent kits (Suzhou Corning Biotechnology Co., Ltd., Suzhou, China). Finally, a portable photosynthesis system (Li-Cor LI-6400, Nebraska, USA) and a chlorophyll fluorescence spectrometer (Heinz Walz GmbH 91090 Effeltrich, Nuremberg, Germany) were employed for the measurement of photosynthetic and chlorophyll fluorescence, respectively.

\subsection{Statistical Analysis}

The data presented are the means of at least three randomized replicates with standard deviations. A variance analysis was done with the SAS/STAT statistical analysis package (v6.12, SAS Institute, Cary, NC, USA).

\section{Conclusions}

PlMYB108, a R2R3-MYB TF, was extracted from P. lactiflora, and its protein was located in the nucleus. The PlMYB108 expression was positively correlated with DT in P. lactiflora. Based on our analysis, PIMYB108 is an essential gene that markedly enhances flavonoid accumulation, ROS scavenging capacity, and photosynthesis and eventually confers DT in tobacco. Our conclusion would provide a theoretical basis for future studies attempting to enhance DS tolerance in P. lactiflora via genetic engineering.

Supplementary Materials: The following are available online at https:/ /www.mdpi.com/article/10 $.3390 /$ ijms222111884/s1.

Author Contributions: Data curation, T.L.; formal analysis, T.L., Y.W., and Z.C.; methodology, T.L. and Y.W.; project administration, J.T. and D.Z.; software, Z.C.; writing-original draft, Y.W. and T.L.; writing-review and editing, Y.W. and T.L. All authors have read and agreed to the published version of the manuscript.

Funding: This work was supported by the National Natural Science Funds (32102411), Modern Agricultural Industrial Technology System in Jiangsu Province (JATS [2020] 436), the Qing Lan Project of Jiangsu Province and the High-Level Talent Support Program of Yangzhou University, the Jiangsu Association for Science and Technology Young Scientific and Technological Talents Project Supported by Yanqing $\mathrm{Wu}$, the Agricultural Science and Technology Independent Innovation Fund of Jiangsu Province (CX [20] 2030, CX [20] 3021), the Natural Science Foundation of Jiangsu Province of China (BK20200924).

Institutional Review Board Statement: Not applicable.

Informed Consent Statement: Not applicable.

Data Availability Statement: Not applicable.

Conflicts of Interest: The authors declare no conflict of interest. 


$\begin{array}{ll}\text { Abbreviations } \\ \text { MYB } & \text { Myeloblastoma } \\ \text { TF } & \text { Transcription factor } \\ \text { DS } & \text { Drought stress } \\ \text { aa } & \text { Amino acids } \\ \text { DT } & \text { Drought tolerance } \\ \text { WT } & \text { Wild-type } \\ \text { ROS } & \text { Reactive oxygen species } \\ \text { bZIP } & \text { Basic leucine zipper } \\ \text { ORF } & \text { Open reading frame } \\ \text { DEGs } & \text { Differential expression genes } \\ \text { RLWC } & \text { Relative leaf water content } \\ \text { RACE } & \text { Rapid-amplification of cDNA ends } \\ \text { DAB } & \text { Diaminobenzidine } \\ \text { qRT-PCR } & \text { Quantitative real-time polymerase chain reaction }\end{array}$

\section{References}

1. Farooq, M.; Wahid, A.; Kobayashi, N.; Fujita, D.; Basra, S.M.A. Plant drought stress: Effects, mechanisms and management. Agron. Sustain. Dev. 2009, 29, 182-212. [CrossRef]

2. He, X.; Xu, L.; Pan, C.; Gong, C.; Wang, Y.; Liu, X.; Yu, Y. Drought resistance of Camellia oleifera under drought stress: Changes in physiology and growth characteristics. PLOS ONE 2020, 15, e0235795. [CrossRef]

3. Dhansu, P.; Kulshreshtha, N.; Kumar, R.; Raja, A.K.; Pandey, S.K.; Goel, V.; Ram, B. Identification of Drought-Tolerant Co-canes Based on Physiological Traits, Yield Attributes and Drought Tolerance Indices. Sugar Tech 2021, 23, 747-761. [CrossRef]

4. Ozturk, A.; Erdem, E.; Aydin, M.; Karaoglu, M.M. The effects of drought after anthesis on the grain quality of bread wheat depend on drought severity and drought resistance of the variety. Cereal Res. Commun. 2021, 3, 1-12. [CrossRef]

5. Zhao, D.Q.; Zhang, X.Y.; Fang, Z.W.; Wu, Y.Q.; Tao, J. Physiological and transcriptomic analysis of tree peony (Paeonia section Moutan DC.) in response to drought stress. Forests 2019, 10, 135. [CrossRef]

6. Ahmed, R.F.; Irfan, M.; Shakir, H.A.; Khan, M.; Chen, L. Engineering drought tolerance in plants by modification of transcription and signalling factors. Biotechnol. Biotechnol. Equip. 2020, 34, 781-789. [CrossRef]

7. Baldoni, E.; Genga, A.; Cominelli, E. Plant MYB Transcription Factors: Their Role in Drought Response Mechanisms. Int. J. Mol. Sci. 2015, 16, 15811-15851. [CrossRef] [PubMed]

8. Leng, P.; Zhao, J. Transcription factors as molecular switches to regulate drought adaptation in maize. Theor. Appl. Genet. 2019, 133, 1455-1465. [CrossRef]

9. Joshi, R.; Wani, S.; Singh, B.; Bohra, A.; Dar, Z.; Lone, A.; Pareek, A.; Singla-Pareek, S.L. Transcription Factors and Plants Response to Drought Stress: Current Understanding and Future Directions. Front. Plant Sci. 2016, 7, 1029. [CrossRef]

10. Riechmann, J.L.; Heard, J.; Martin, G.; Reuber, L.; Jiang, C.-Z.; Keddie, J.; Adam, L.; Pineda, O.; Ratcliffe, O.J.; Samaha, R.R.; et al. Arabidopsis Transcription Factors: Genome-Wide Comparative Analysis Among Eukaryotes. Science 2000, 290, 2105-2110. [CrossRef]

11. Stracke, R.; Werber, M.; Weisshaar, B. The R2R3-MYB gene family in Arabidopsis thaliana. Curr. Opin. Plant Biol. 2001, 4, 447-456. [CrossRef]

12. Prouse, M.B.; Campbell, M. The interaction between MYB proteins and their target DNA binding sites. Biochim. Biophys. Acta (BBA)-Bioenerg. 2012, 1819, 67-77. [CrossRef] [PubMed]

13. Bilaud, T.; Koering, C.E.; Binet-Brasselet, E.; Ancelin, K.; Pollice, A.; Gasser, S.M.; Gilson, E. The telobox, a Myb-related telo-meric DNA binding motif found in proteins from yeast, plants and human. Nucleic Acids Res. 1996, 24, 1294-1303. [CrossRef] [PubMed]

14. Zhou, C.G.; Li, C.H. A novel R2R3-MYB transcription factor BpMYB106 of birch (Betula platyphylla) confers increased photosynthesis and growth rate through up-regulating photosynthetic gene expression. Front. Plant Sci. 2016, 7, 315. [CrossRef]

15. Zhang, C.-Y.; Liu, H.-C.; Zhang, X.-S.; Guo, Q.-X.; Bian, S.-M.; Wang, J.-Y.; Zhai, L.-L. VcMYB4a, an R2R3-MYB transcription factor from Vaccinium corymbosum, negatively regulates salt, drought, and temperature stress. Gene 2020, 757, 144935. [CrossRef] [PubMed]

16. Wei, Q.; Chen, R.; Wei, X.; Liu, Y.; Zhao, S.; Yin, X.; Xie, T. Genome-wide identification of R2R3-MYB family in wheat and functional characteristics of the abiotic stress responsive gene TaMYB344. BMC Genom. 2020, 21, 792. [CrossRef]

17. Zhang, L.; Zhao, G.; Xia, C.; Jia, J.; Liu, X.; Kong, X. A wheat R2R3-MYB gene, TaMYB30-B, improves drought stress tolerance in transgenic Arabidopsis. J. Exp. Bot. 2012, 63, 5873-5885. [CrossRef] [PubMed]

18. Fang, Q.; Wang, X.; Wang, H.; Tang, X.; Liu, C.; Yin, H.; Ye, S.; Jiang, Y.; Duan, Y.; Luo, K. The poplar R2R3 MYB transcription factor PtrMYB94 coordinates with abscisic acid signaling to improve drought tolerance in plants. Tree Physiol. 2019, 40, 46-59. [CrossRef]

19. Wang, Q.; Zhao, R.; Chen, Q.H.; da Silva, J.A.T.; Chen, L.Q.; Yu, X.N. Physiological and biochemical responses of two herbaceous peony cultivars to drought stress. Hortscience 2019, 54, 492-498. [CrossRef] 
20. Li, T.; Wang, R.; Zhao, D.; Tao, J. Effects of drought stress on physiological responses and gene expression changes in herbaceous peony (Paeonia lactiflora Pall.). Plant Signal. Behav. 2020, 15, 1746034. [CrossRef]

21. Yang, X.Y.; Lu, M.Q.; Wang, Y.F.; Wang, Y.R.; Liu, Z.J.; Chen, S. Response Mechanism of Plants to Drought Stress. Horticulturae 2021, 7, 50. [CrossRef]

22. Wang, S.; Shi, M.; Zhang, Y.; Xie, X.; Sun, P.; Fang, C.; Zhao, J. FvMYB24, a strawberry R2R3-MYB transcription factor, improved salt stress tolerance in transgenic Arabidopsis. Biochem. Biophys. Res. Commun. 2021, 569, 93-99. [CrossRef] [PubMed]

23. Zhang, Q.; Hao, R.; Xu, Z.; Yang, W.; Wang, J.; Cheng, T.; Pan, H.; Zhang, Q. Isolation and functional characterization of a R2R3-MYB regulator of Prunus mume anthocyanin biosynthetic pathway. Plant Cell Tissue Organ Cult. 2017, 131, 417-429. [CrossRef]

24. Mandaokar, A.; Browse, J. MYB108 acts together with MYB24 to regulate jasmonate-mediated stamen maturation in Arabidopsis. J. Plant Physiol. 2009, 149, 851-862. [CrossRef] [PubMed]

25. Qin, Y.; Wang, M.; Tian, Y.; He, W.; Han, L.; Xia, G. Over-expression of TaMYB33 encoding a novel wheat MYB transcription factor increases salt and drought tolerance in Arabidopsis. Mol. Biol. Rep. 2012, 39, 7183-7192. [CrossRef]

26. Wu, J.; Jiang, Y.; Liang, Y.; Chen, L.; Chen, W.; Cheng, B. Expression of the maize MYB transcription factor ZmMYB3R enhances drought and salt stress tolerance in transgenic plants. Plant Physiol. Biochem. 2019, 137, 179-188. [CrossRef]

27. Zhang, S.; Zhao, Q.C.; Zeng, D.X.; Xu, J.H.; Zhou, H.G.; Wang, F.L.; Ma, N.; Li, Y.H. RhMYB108, an R2R3-MYB transcription factor, is involved in ethylene- and JA-induced petal senescence in rose plants. Hortic. Res. 2019, 6, 139. [CrossRef]

28. Zhou, M.P.; Zhou, X.Q.; Yao, J.B.; Zhang, Z.Y.; Zhang, P.; Yang, X.M.; Ma, H.X. Preliminary analysis of drought tolerance in MYB transgenic wheat. Jiangsu J. Agr. Sci. 2013, 29, 474-479. (In Chinese)

29. Nakabayashi, R.; Yonekura-Sakakibara, K.; Urano, K.; Suzuki, M.; Yamada, Y.; Nishizawa, T.; Matsuda, F.; Kojima, M.; Sa-kakibara, H.; Shinozaki, K. Enhancement of oxidative and drought tolerance in Arabidopsis by overaccumulation of antioxidant flavonoids. Plant J. 2014, 77, 367-739. [CrossRef]

30. Zhao, D.Q.; Luan, Y.T.; Shi, W.B.; Zhang, X.Y.; Meng, J.S.; Tao, J. A Paeonia ostii caffeoyl-CoA O-methyltransferase confers drought stress tolerance by promoting lignin synthesis and ROS scavenging. Plant Sci. 2021, 303, 110765. [CrossRef] [PubMed]

31. Zhao, D.; Shi, W.; Xia, X.; Tang, Y.; Tao, J. Microstructural and lignin characteristics in herbaceous peony cultivars with different stem strengths. Postharvest Biol. Technol. 2019, 159, 111043. [CrossRef]

32. Zhao, D.Q.; Xia, X.; Su, J.H.; Wei, M.R.; Wu, Y.Q.; Tao, J. Overexpression of herbaceous peony HSP70 confers high temperature tolerance. BMC Genom. 2019, 20, 70. [CrossRef] [PubMed] 